\title{
Professor Mikio Sato and Microlocal Analysis
}

\author{
by \\ Masaki KASHIWARA and Takahiro KAWAI
}

\begin{abstract}
We describe the impact of microlocal analysis on mathematical sciences and the role Prof. Mikio Sato played in its creation and development.
\end{abstract}

2010 Mathematics Subject Classification: 32C38, 35A27, 32A45, 14F10, 13 N10.

Keywords: microlocal analysis, hyperfunctions, microfunctions.

\section{$\S 1$. The summer of 1957}

In the summer of 1957, Sato created the theory of hyperfunctions. In formulating the idea of regarding boundary values of holomorphic functions as generalized functions, he introduced the notion of relative cohomology groups, independently of A. Grothendieck. The sheaf of hyperfunctions was thus constructed without using the space of $\mathrm{C}^{\infty}$-functions and its dual space, with which Sato did not feel happy. Furthermore it is an ultimate object as a generalization of functions in the sense that it is a flabby sheaf. It was the first example that visualized algebraic analysis of our age. The full account of the theory of hyperfunctions was published in 1959 and 1960 ([6]).

\section{§2. June 24, 1960}

Shortly before his departure for the Institute for Advanced Study (IAS) at Princeton, Sato gave a colloquium talk entitled "On systems of linear partial differential equations" at the University of Tokyo, where he described his program to develop analysis through investigation of the structure of differential equations. The notes

This is a contribution to the special issue "The golden jubilee of algebraic analysis".

Communicated by S. Mukai. Received September 13, 2010.

M. Kashiwara, T. Kawai: Research Institute for Mathematical Sciences, Kyoto University, Kyoto, 606-8502, Japan;

e-mail: masaki@kurims.kyoto-u.ac.jp 
of this talk taken by H. Komatsu are reproduced in this issue (pp. 2-9). In his talk, Sato emphasized grasping a system of linear partial differential equations as a $\mathscr{D}$-module, where $\mathscr{D}$ denotes the sheaf of differential operator: ${ }^{1}$, and then studying the structure of its solutions through the cohomological machinery; Hom $\mathscr{D}$ describes its solutions, $\mathrm{Ext}_{\mathscr{D}}^{1}$ describes the obstructions to its solvability, etc. He further pointed out that important and basic objects such as Riemann functions for hyperbolic equations, i.e., elementary solutions, should be controlled by a kind of strongly overdetermined systems, which later became called maximally overdetermined systems or holonomic systems.

\section{$\S 3$. Interlude-from 1960 to 1968}

Although Sato's talk in June 1960 was a challenging and inspiring one, it remained, in all honesty, a "frame without a picture" for a while, with the exception of H. Komatsu's work ([4]) on the structure of hyperfunction solutions of systems of linear partial differential equations with constant coefficients. Apparently influenced by the atmosphere of IAS, Sato himself seemed to be totally absorbed in number theory, as is mentioned in the Preface of this issue. Thanks to the efforts of H. Komatsu, the Sato-Komatsu seminar began in 1968 at the University of Tokyo, after a course by Komatsu entitled "Hyperfunctions and linear partial differential equations with constant coefficients" given in the academic year 1967 (from April 1967 through February 1968). Sato's interest in differential equations was gradually resurrected by the discussions with H. Komatsu and his students who attended the seminar.

\section{§4. March, 1969}

Having been thinking over the structure of hyperfunctions in conjunction with the edge of the wedge theorem which had originated from quantum field theory, Sato realized in March of 1969 that singularities of hyperfunctions could be dispersed on the cotangent bundle; that is, we can construct the sheaf $\mathscr{C}_{M}$ of microfunctions on the spherical cotangent bundle $\sqrt{-1} S^{*} M$ so that the following exact sequence holds:

$$
0 \rightarrow \mathscr{A}_{M} \rightarrow \mathscr{B}_{M} \stackrel{\mathrm{sp}}{\longrightarrow} \pi_{*} \mathscr{C}_{M} \rightarrow 0
$$

where $\mathscr{A}_{M}$ (resp. $\mathscr{B}_{M}$ ) denotes the sheaf of real analytic functions on a real analytic manifold $M$ (resp. hyperfunctions on $M$ ) and $\pi_{*}$ signifies the 0 -th direct image by the canonical projection $\pi: \sqrt{-1} S^{*} M \rightarrow M$. Furthermore an $m$-th order linear

\footnotetext{
${ }^{1}$ The German letter $\mathfrak{T}$ is used instead of $\mathscr{D}$ in the notes.
} 
differential equation

$$
P u=f, \quad \text { for a given function } f \in \mathscr{A}_{M},
$$

entails

$$
\operatorname{supp}(\operatorname{sp}(u)) \subset\left\{(x, \sqrt{-1} \xi) \in \sqrt{-1} S^{*} M ; p_{m}(x, \sqrt{-1} \xi)=0\right\},
$$

where $p_{m}$ denotes the principal symbol of the $m$-th order operator $P$. This genesis of microfunctions marked the dawn of microlocal analysis $([7,[8]$ ).

\section{$\S 5$. October, 1971}

The years 1970 and 1971 were the golden age of microlocal analysis, and the core of the results was reported at the Katata conference held in October, 1971, and 12 is its report. In view of the introduction of the sheaf of microfunctions, Sato and his collaborators established the following scheme in the study of linear differential equations: instead of studying differential equations and their hyperfunction solutions on a given manifold $M$, study the corresponding microdifferential equations and their microfunction solutions. This scheme further enhanced a viewpoint stated in the 1960 talk: study the structure of $\mathscr{E}_{X}$-modules, where $\mathscr{E}_{X}$ designates the sheaf of microdifferential operators ${ }^{2}$ on the projective cotangent bundle $P^{*} X$ of the complexification $X$ of $M$. Furthermore we can apply a quantized contact transformation $\Phi$ to microdifferential operators associated to the contact transformation $\varphi$ on $P^{*} X$ (cf. Egorov [1, Hörmander [2]). Using quantized contact transformations of microdifferential operators we obtain the following decisive Structure Theorems 1, 2 and 3. In a word they claim: the microlocal structure of a microdifferential equation $\mathscr{M}$, and hence that of its microfunction solutions, is described by the characteristic variety $V:=\operatorname{Supp}(\mathscr{M})$ at its generic point.

Structure Theorem 1. Let $\mathscr{M}$ be a coherent left $\mathscr{E}_{X}$-module and let $V$ denote $\operatorname{Supp}(\mathscr{M})$. Suppose that

$$
\mathscr{E}_{x t_{\mathscr{E}_{X}}^{j}}\left(\mathscr{M}, \mathscr{E}_{X}\right)=0 \text { for } j \neq d
$$

holds for some integer $d$, and suppose further that $V$ is regular at a point $p$ in the sense that $V$ is non-singular on a neighborhood of $p$ and that

$$
\left.\omega\right|_{V}(p) \neq 0
$$

${ }^{2} \mathrm{~A}$ comment on the terminology and notation used in [12: a microdifferential operator is called a pseudo-differential operator and the symbol $\mathcal{P}$ is used instead of $\mathscr{E}$ in 12 . Further the symbol $\mathcal{P}^{f}$ is used to designate the sheaf of finite (versus infinite) order pseudo-differential operators. In recent literature, the couple of symbols $(\mathscr{E}, \mathscr{E} \infty)$ is more commonly used than $\left(\mathcal{P}^{f}, \mathcal{P}\right)$, and hence we use $(\mathscr{E}, \mathscr{E} \infty)$ instead of $\left(\mathcal{P}^{f}, \mathcal{P}\right)$ in what follows. 
for the canonical 1-form $\omega$ of $P^{*} X$. Then after an appropriate quantized contact transformation $(\Phi, \varphi), \mathscr{E}_{X}^{\infty} \otimes_{\mathscr{E}_{X}} \mathscr{M}$ is isomorphic to a direct summand of a finite direct sum of the partial de Rham system $\mathscr{N}_{0}$ where

$$
\mathscr{N}_{0}=\mathscr{E}_{\mathbb{C}^{n}}^{\infty} /\left(\sum_{j=1}^{d} \mathscr{E}_{\mathbb{C}^{n}}^{\infty} \frac{\partial}{\partial z_{j}}\right)
$$

and the contact transformation $\varphi$ satisfies $\varphi(p)=(0,(0,0, \ldots, 0,1)) \in P^{*} \mathbb{C}^{n}$.

Through the study of the canonical form of $V$ under real contact transformations, Structure Theorem 1 entails the following result on $\sqrt{-1} S^{*} M$.

Structure Theorem 2. Let $X$ be the complexification of a real analytic manifold $M$, and let $\mathscr{M}$ be the coherent $\mathscr{E}_{X}$-module studied in Structure Theorem 1. For a point $p \in V \cap \sqrt{-1} S^{*} M$ suppose

$$
V \cap \bar{V} \text { is regular at } p \text {, }
$$

and

$$
T_{q} V \cap T_{q} \bar{V}=T_{q}(V \cap \bar{V})
$$

for any $q \in V \cap \bar{V}$ that is sufficiently close to $p$. Here $\bar{V}$ denotes the complex conjugate of $V$ (with respect to $\sqrt{-1} S^{*} M$ ) and $T_{q}(V)$ etc. stand for the tangent space of $V$ etc. at $q$. Further suppose that the generalized Levi form ${ }^{3} L_{V}$ associated with $V$ has a constant signature $(a, b)$ near $p$.

Then after a suitable real contact transformation, $\mathscr{E}_{X}^{\infty} \otimes_{\mathscr{E}_{X}} \mathscr{M}$ is isomorphic to a direct summand of $\mathscr{E}_{X}^{\infty} \otimes_{\mathscr{E}_{X}} \mathscr{N}$, where $\mathscr{N}$ is the following system of differential equations defined near $(x, \sqrt{-1} \xi)=(0, \sqrt{-1}(0,0, \ldots, 0,1)) \in \sqrt{-1} S^{*} \mathbb{R}^{n}$ :

$$
\left\{\begin{array}{l}
\frac{\partial u}{\partial x_{j}}=0 \quad \text { for } j=1, \ldots, r \\
\left(\frac{\partial}{\partial x_{r+2 k-1}}+\sqrt{-1} \frac{\partial}{\partial x_{r+2 k}}\right) u=0 \quad \text { for } k=1, \ldots, s \\
\left(\frac{\partial}{\partial x_{r+2 s+l}}+\sqrt{-1} x_{r+2 s+l} \frac{\partial}{\partial x_{n}}\right) u=0 \quad \text { for } l=1, \ldots, a, \\
\left(\frac{\partial}{\partial x_{r+2 s+l}}-\sqrt{-1} x_{r+2 s+l} \frac{\partial}{\partial x_{n}}\right) u=0 \quad \text { for } l=a+1, \ldots, a+b
\end{array}\right.
$$

\footnotetext{
${ }^{3}$ With the expression $V=\bigcap_{j=1}^{d} g_{j}^{-1}(0)$, the generalized Levi form at $q$ means the Hermitian form given by $L_{V}\left(v_{1}, \ldots, v_{d}\right)=\sum_{1<j, k<d}\left\{g_{j}, \overline{g_{k}}\right\}(q) v_{j} \overline{v_{k}}$ where $\left\{g_{j}, \overline{g_{k}}\right\}$ denotes the Poisson bracket of $g_{j}$ and $\overline{g_{k}}$.
} 
where

$$
\begin{aligned}
& r=\operatorname{codim} V-\operatorname{codim}(V \cap \bar{V}), \\
& s=\operatorname{codim} V-(a+b+r) .
\end{aligned}
$$

Once we obtain Structure Theorem 2, the structure of microfunction solutions of the system $\mathscr{M}$ can be easily obtained.

Structure Theorem 3. Let $(M, X, \mathscr{M}, V, p)$ be as in Structure Theorem 2. Then

$$
\mathscr{E}_{x} t_{\mathscr{E}_{X}}^{j}\left(\mathscr{M}, \mathscr{C}_{M}\right)=0 \text { for } j \neq a
$$

Furthermore the remaining sheaf

$$
\mathcal{F}:=\mathscr{E}_{x} t_{\mathscr{E}_{X}}^{a}\left(\mathscr{M}, \mathscr{C}_{M}\right) \simeq \mathscr{E}_{x} t_{\mathscr{E}_{X}^{\infty}}^{a}\left(\mathscr{E}_{X}^{\infty} \otimes_{\mathscr{E}_{X}} \mathscr{M}, \mathscr{C}_{M}\right)
$$

has the following structure on a neighborhood $U$ of $p$ : there exist an s-dimensional complex manifold $Y$, a real analytic manifold $N$, a map

$$
\varphi: V \cap U \cap \sqrt{-1} S^{*} M \rightarrow Y \times \sqrt{-1} S^{*} N
$$

and a sheaf $\mathcal{G}$ on $Y \times \sqrt{-1} S^{*} N$ such that

$$
\mathcal{F} \simeq \varphi^{-1} \mathcal{G}
$$

Moreover $\mathcal{G}$ is a direct summand of a finite direct sum of a solution sheaf of the Cauchy-Riemann equation associated with $Y$.

Thus the microlocal structure of microfunction solutions of a general system of microdifferential equations is completely clarified.

As for maximally overdetermined systems, the condition 5.2 is not satisfied as a consequence of their "maximal" character. Thus the above Structure Theorems cannot be applied. Instead, [12] developed the theory of maximally overdetermined (= holonomic) systems separately. It introduced a basic invariant "order" for a simple holonomic system and investigated its behavior under various operations such as integrations, products and restrictions.

Thus the "frame" that Sato proposed in 1960 was filled up by an impressively spectacular picture.

\section{§6. September, 1972}

The use of infinite-order operators is crucial in Structure Theorem 1 in the previous section. The so-called constant multiplicity (of the characteristic variety) problem 
is circumvented by the aid of infinite-order microdifferential operators. But operators of infinite order played only an auxiliary role there; the starting equation is a finite-order one, and its canonical form is also of finite order. Concerning the importance of infinite-order operators, Sato [9] made a challenging proposal at the Orsay conference held in September, 1972: Use infinite-order equations to characterize wild functions such as the theta zero-values. This proposal has been put into practice in [11]. By way of parenthesis we note that the phrase "micro-local analysis" first appeared in the proceedings of the Orsay conference (Astérisque 2 et $3(1973)$ ).

\section{§7. January, 1975}

Sato stayed in Nice during the academic year 1972, and he had the chance of discussing the analytic properties of the $S$-matrix with F. Pham there. Through the discussion with Pham, Sato realized that the analysis of the $S$-matrix done by Iagolnitzer-Stapp (3]) could be regarded as a prototype of the theory of microfunctions. Encouraged by this observation, he ([10]) proposed at a conference on mathematical physics (Kyoto, January 1975) to study Green's function in quantum field theory and related functions from the viewpoint of microlocal analysis and holonomic systems, having in mind correlation functions for the 1-dimensional Ising model as a toy. This fact seems to have foreshadowed his works on the 2dimensional Ising model, which are described by McCoy [5] in this issue. Let us now hand over the baton to Prof. McCoy.

\section{References}

[1] Ju. V. Egorov, The canonical transformations of pseudodifferential operators, Uspekhi Mat. Nauk 24 (1969), no. 5 (149), 235-236 (in Russian). Zbl 0191.43802 MR 0265748

[2] L. Hörmander, Fourier integral operators I, Acta Math. 127 (1971), 79-183. Zbl 0212.46601 MR 0388463

[3] D. Iagolnitzer and H. P. Stapp, Macroscopic causality and physical region analyticity in S-matrix theory, Comm. Math. Phys. 14 (1969), 15-55. MR 0253651

[4] H. Komatsu, Resolutions by hyperfunctions of sheaves of solutions of differential equations with constant coefficients, Math. Ann. 176 (1968), 77-86. Zbl 0161.29802 MR 0226181

[5] B. M. McCoy, Mikio Sato and mathematical physics, this issue.

[6] M. Sato, Theory of hyperfunctions I, II, J. Fac. Sci. Univ. Tokyo Sect. IA 8 (1959/1960),

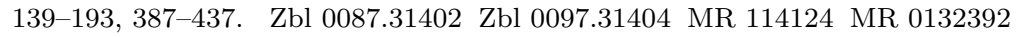

[7] Hyperfunctions and partial differential equations, in Proc. Internat. Conf. on Functional Analysis and Related Topics (Tokyo, 1969), Univ. Tokyo Press, Tokyo, 1970, 91-94. Zbl 0208.35801

[8] Regularity of hyperfunction solutions of partial differential equations, in Actes du Congrès Internat. Math., Tome 2, Gauthier-Villars, Paris, 1971, 785-794. Zbl 0232.35004 MR 0650826 
[9] _ Pseudo-differential equations and theta functions, Astérisque 2 et 3 (1973), 286291. Zbl 0288.35045 MR 0467850

[10] _ Recent development in hyperfunction theory and its applications to physics (microlocal analysis of $S$-matrices and related quantities), in Internat. symposium on mathematical problems in theoretical physics (Kyoto, 1975), Lecture Notes in Phys. 39, Springer, 1975, 13-29. Zbl 0331.46033 MR 0650988

[11] M. Sato, M. Kashiwara and T. Kawai, Microlocal analysis of theta functions, in Group representations and systems of differential equations (Tokyo, 1982), Adv. Stud. Pure Math. 4, Kinokuniya, Tokyo, 1984, 267-289. Zbl 0603.10023 MR 0810634

[12] M. Sato, T. Kawai and M. Kashiwara, Microfunctions and pseudo-differential equations, in Hyperfunctions and pseudo-differential equations (Katata, 1971), Lecture Notes in Math. 287, Springer, 1973, 265-529. Zbl 0277.46039 MR 0420735 